\title{
Quantitative determination of hazardous substances in aerosols by light scattering and machine learning with the example of $\mathrm{Cr}(\mathrm{VI})$ in electroplating processes
}

\author{
Andreas Peckhaus ${ }^{1 *}$, Arne Walter ${ }^{2}$, Marian Kraus ${ }^{2}$, Florian Gebert ${ }^{2}$ and Frank Duschek ${ }^{2}$ \\ 1 German Aerospace Center (DLR), Institute of Technical Physics, Stuttgart, Pfaffenwaldring 38-40, 70569 Stuttgart \\ 2 German Aerospace Center (DLR), Institute of Technical Physics, Lampoldshausen, Langer Grund, 74239 Hardthausen \\ *andreas.peckhaus@dlr.de
}

\begin{abstract}
Regulations of safety usage of hazardous substances impose companies to monitor their emissions. A novel approach determines the mass concentration of $\mathrm{Cr}(\mathrm{VI})$ in exhaust airflow based on angular light scattering combined with machine learning algorithms.

(C) 2021 The Author(s)
\end{abstract}

\section{Introduction}

The regulation (EC) No. 1907/2006 (REACH) addresses the proper handling of chemical substances in the European Union with the objective to protect human life and the environment against hazardous substances [1]. However, the regulation imposes currently a tremendous burden to the industry. Hazardous substances, like the chromium(VI) oxide, were included in the annex XVII of the regulation, with the consequence that industrial companies using $\mathrm{Cr}(\mathrm{VI})$ in their electroplating processes must establish standards for worker and environment protection or find substitute substances (e.g. Cr(III)). Likewise in the USA, the Environmental Protection Agency (EPA) has defined a list of hazardous air pollutants including $\mathrm{Cr}(\mathrm{VI})$ as a particulate matter [2].

During the electroplating process, a mist of bubbling electrolyte solution above the bath is formed which is removed by a fume hood. Wet scrubbers remove only large aerosol particles $(>5 \mu \mathrm{m})$, but smaller respirable particles are still present in the exhaust air. In addition, wet scrubbers introduce water particles. Current measurement techniques are not capable of monitoring the mass concentration of $\mathrm{Cr}(\mathrm{VI})$ in real-time. Particles from the exhaust airflow are collected on a filter element for several hours. The filter residues are eluted and converted into an optically active complex and the $\mathrm{Cr}(\mathrm{VI})$ content is determined photometrically. A similar technique uses a water-filled wash bottle for collection. The solution is separated via chromatography and $\mathrm{Cr}(\mathrm{VI})$ is detected by a UV-VIS spectrometer [3]. The disadvantages of these techniques are that they are very time-consuming, not suited for on-line monitoring of electroplating processes and use further chemicals for the analysis.

To improve the monitoring of $\mathrm{Cr}(\mathrm{VI})$-containing particles in the exhaust airflow a novel approach is presented. The angular light scattering from $\mathrm{Cr}(\mathrm{VI})$-containing particles and water particles is used in combination with machine learning algorithms to distinguish between particle types and to determine the mass concentration of $\mathrm{Cr}(\mathrm{VI})$ in the exhaust airflow. With this approach, anomalies can be recognized at an early stage which may indicate problems in the industrial process, like flawed plating.

\section{Concept and model}

To evaluate the feasibility of the single-particle scattering approach, a detection system and an aerosol with particles of different sizes and compositions are defined. For the aerosol, two particle compositions, pure water, and an electrolyte for chrome plating are assumed. For each particle composition and laser wavelength, angle-resolved scattering intensities have been calculated for scattering angles from $1^{\circ}$ to $179^{\circ}$ and particle diameters from $0.16 \mu \mathrm{m}$ to $26.43 \mu \mathrm{m}$ based on Lorenz-Mie theory [4]. These data sets are used to test the performance of different system setups with respect to the combination of laser wavelengths and detection angles.

The calculated scattered intensities are distorted with three types of artificial sources of noise. Dark current-like background noise, noise due to laser power fluctuations and miscellaneous noise due to detector efficiency are assumed. For each combination of the detection angles $\theta_{1}$ and $\theta_{2}$ of each system configuration (see Fig.1), the resulting data set with four features is randomly divided into two parts. $60 \%$ is used as training data and the remaining $40 \%$ is used to test the performance of the trained models. The C5.0 decision tree algorithm is used for particle discrimination $[5,6]$. Furthermore, for each set of detection angles a regression model is generated using random forests to predict the size of the $\mathrm{Cr}(\mathrm{VI})$-containing particles. The accuracy of discrimination and the coefficient of determination $\left(\mathrm{R}^{2}\right)$ are calculated for each system configuration. 


\section{Results}

Based on high values for the accuracy of discrimination and $\mathrm{R}^{2}$ for size determination, an optimal arrangement of the detection system is proposed $\left(\theta_{1}=85^{\circ}\right.$ and $\left.\theta_{2}=140^{\circ}\right)$ for the generation of classification and regression models. For a new data set, the particle masses are calculated and summed up. Assuming a constant aerosol concentration, the amount of hazardous material in the exhaust airflow is obtained. The right panel of Fig.1 shows the estimated masses and the predicted masses of particles classified as chromate particles.

According to the classification of particles, the following conclusions can be drawn: The accuracy of classification is reduced when both detectors are positioned in the forward scattering direction $\left(0-60^{\circ}\right)$. At these angles, the scattering intensity is largely independent of the particle's refractive index. For parallel polarization $\left(\varphi=0^{\circ}\right)$, the accuracy decreases if one detection angle is chosen to be in the range from $90^{\circ}$ to $120^{\circ}$. This effect can be seen for all combinations but is generally less severe for shorter wavelengths. Contrary to classification, the particle size determination by regression yields good results for forward scattering for both polarizations. For parallel polarization, the presence of the so-called pseudo-Brewster angle complicates the regression analysis. The pseudo-Brewster angle is located at around $70^{\circ}$. The effect is more pronounced for larger and weakly absorbing $\mathrm{Cr}(\mathrm{VI})$-containing particles than for water particles. Both classification and regression are combined to retrieve the accuracy of the volume estimation (i.e. the mass concentration of $\mathrm{Cr}(\mathrm{VI}))$ for different system configurations.
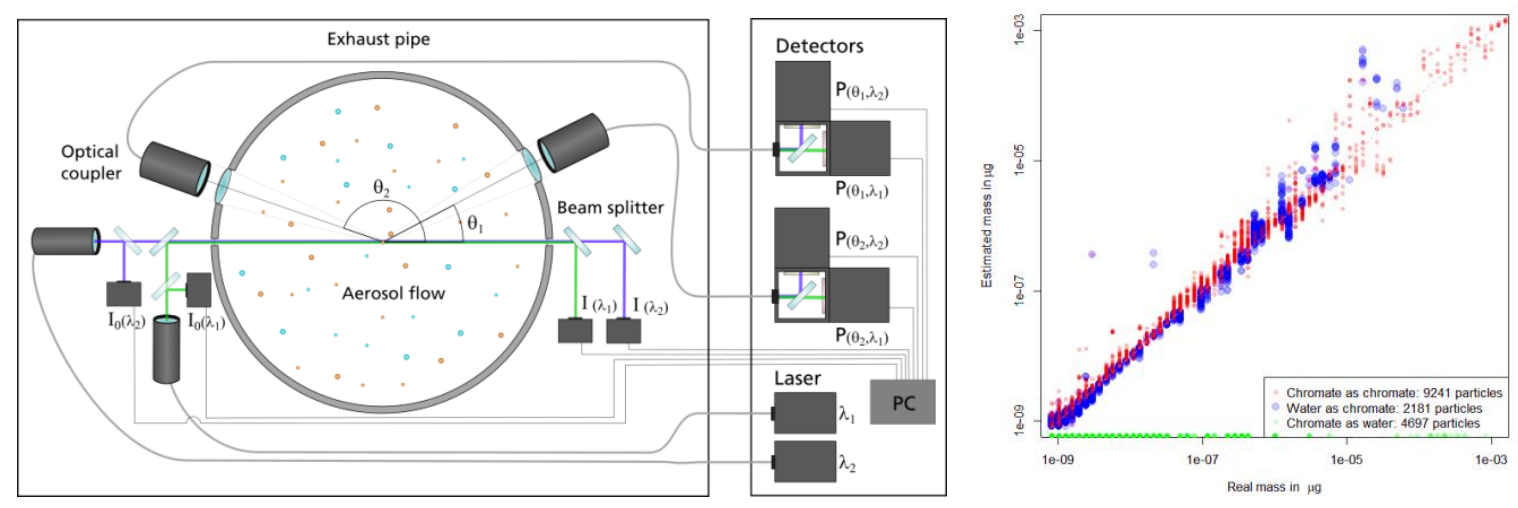

Fig. 1. Proposed setup for the detection system (left) and predicted masses of chromate particles in a simulated aerosol consisting of water and chromate particles (right). The following model parameters are assumed: wavelengths: $405 / 520 \mathrm{~nm}$, polarization: $0^{\circ}$, detector noise: $50 \%$, laser noise: $10 \%$, random noise: $10 \%$, detection angles: $85 / 140^{\circ}$. The real and estimated chromate masses are $0.116 \mu \mathrm{g}$ and $0.088 \mu \mathrm{g}$, respectively. This results in a prediction of $75.38 \%$ chromate.

\section{References}

[1] https://eur-lex.europa.eu/legal-content/DE/TXT/PDF/?uri=CELEX:02006R1907-20140410\&from=DE

[2] https://www3.epa.gov/ttnamti1/files/ambient/airtox/workbook/AirToxicsWorkbook6-09.pdf

[3] https://publikationen.dguv.de/regelwerk/regelwerk-nach-fachbereich/rohstoffe-und-chemische-industrie/gefahrstoffe/187/verfahren-zur bestimmung-von-sechswertigem-chrom

[4] Bohren, C. F. \& Huffman, D. R., Absorption and Scattering of Light by Small Particles, John Wiley \& Sons, Inc., 1983.

[5] Kuhn M, Quinlan R, C50: C5.0 Decision Trees and Rule-Based Models, 2018. Available at https://CRAN.R-project.org/package=C50

[6] R Core Team. R: A language and environment for statistical computing. R Foundation for Statistical Computing, Vienna, Austria, 2014. Available at: http://www.R-project.org/ 